\title{
Genome-wide association study reveals genetic loci and candidate genes for average daily gain in Duroc pigs
}

\author{
Jianping Quan ${ }^{1, a}$, Rongrong Ding ${ }^{1, a}$, Xingwang Wang ${ }^{1}$, Ming Yang ${ }^{2}$, Yang Yang ${ }^{1}$, Enqin Zheng ${ }^{1}$, Ting Gu ${ }^{1}$, \\ Gengyuan $\mathrm{Cai}^{2}$, Zhenfang Wu ${ }^{1,2}$, Dewu Liu, ${ }^{1, *}$, and Jie Yang ${ }^{1, *}$
}

\author{
* Corresponding Authors: \\ Dewu Liu \\ Tel: +86-13640835778, Fax: +86-020-85284816, \\ E-mail: dwliu@scau.edu.cn \\ Jie Yang \\ Tel: +86-18602020087, Fax: +86-020-85283780, \\ E-mail: jieyang2012@hotmail.com
}

${ }^{1}$ College of Animal Science and National Engineering Research Center for Breeding Swine Industry, South China Agricultural University, Guangzhou 510642 China

${ }^{2}$ National Engineering Research Center for Breeding Swine Industry, Guangdong Wens Foodstuffs Co., Ltd, Yunfu 527400, China

a These authors contributed equally to this work.

ORCID

Dewu Liu

https://orcid.org/0000-0002-2234-0861

Jie Yang

https://orcid.org/0000-0002-7031-2160

Submitted May 9, 2017; Revised Aug 8, 2017; Accepted Oct 9, 2017
Objective: Average daily gain (ADG) is an important target trait of pig breeding programs. We aimed to identify single nucleotide polymorphisms (SNPs) and genomic regions that are associated with ADG in the Duroc pig population.

Methods: We performed a genome-wide association study involving 390 Duroc boars and by using the PorcineSNP60K Beadchip and two linear models.

Results: After quality control, we detected 3,5971 SNPs, which included seven SNPs that are significantly associated with the ADG of pigs. We identified six quantitative trait loci (QTL) regions for ADG. These QTLs included four previously reported QTLs on Sus scrofa chromosome (SSC) 1, SSC5, SSC9, and SSC13, as well as two novel QTLs on SSC6 and SSC16. In addition, we selected six candidate genes (general transcription factor $3 \mathrm{C}$ polypeptide 5 , high mobility group AT-hook 2, nicotinamide phosphoribosyltransferase, oligodendrocyte transcription factor 1, pleckstrin homology and RhoGEF domain containing G4B, and ENSSSCG00000031548) associated with ADG on the basis of their physiological roles and positional information. These candidate genes are involved in skeletal muscle cell differentiation, diet-induced obesity, and nervous system development.

Conclusion: This study contributes to the identification of the casual mutation that underlies QTLs associated with ADG and to future pig breeding programs based on marker-assisted selection. Further studies are needed to elucidate the role of the identified candidate genes in the physiological processes involved in $\mathrm{ADG}$ regulation.

Keywords: Genome-wide Association Study (GWAS); Single Nucleotide Polymorphism (SNP); Average Daily Gain; Duroc Pigs

\section{INTRODUCTION}

The pig (Sus scrofa) is an economically important farm animal, and the Duroc $\times$ (Landracex Yorkshire) three-way cross hybrid (DLY) pig is the most popular commercial pig breed worldwide. The growth performance of DLY pigs is directly affected by that of their terminal male parent, the Duroc pig. Growth performance in commercial swine productions systems is measured on the basis of numerous economically important production traits, among which the average daily gain (ADG) is the most important. Pigs with higher ADG can achieve the target market weight within a shorter period than those with lower ADG, thus saving considerable feeding costs. Therefore, pig farmers have aimed to increase the ADG of swine to improve profitability. Fortunately, molecular technologies have helped improve the ADG of commercial swine. The association of several major genes, such as translation elongation factor 1 and corticotropin releasing hormone, and quantitative trait loci (QTLs) with ADG has been confirmed; breeding on the basis of these genes and QTLs might result in the considerable improvement of the ADG of commercial pig herds in the near future [1-4]. 
Previous studies have utilized microsatellite markers to identify the QTLs or major functional genes of quantitative traits, such as ADG $[2,5]$. These QTLs, however, are based on linkage analyses and map construction with few genetic markers, resulting in large QTL spans. QTL spans could be shortened and novel associated variants could be identified via genome-wide association (GWA) studies through the use of recently developed high-throughput commercial genotyping platforms and single nucleotide polymorphisms (SNPs) [6,7]. Thousands of QTLs that control a wide range of pig traits have been identified, and 563 QTLs for ADG have been deposited in PigQTLdb (http://www.animalgenome.org/cgi-bin/QTLdb/ SS/summary). Nevertheless, only a handful of causative genes have been identified via QTL mapping analysis because QTLs span large chromosomal regions that, in turn, comprise hundreds of genes [8]. Therefore, minimizing the QTL region would facilitate the discovery of causative genes.

GWA is a powerful strategy for the genetic dissection of trait loci in humans and other animals $[9,10]$. In this method, high-density molecular markers screened from genomes are used to analyze the association between phenotypic traits and marker data. GWA was first proposed by Risch et al [11]. More than 60,000 SNPs can be analyzed with high efficiency following the development of high-throughput commercial genotyping platforms for pigs [12]. Consequently, this development has enabled the realistic estimation of genetic diversity and genome structure [13]. In addition, more than one model can be used for GWA analysis [14,15].

In this study, we subjected the genomic data of 390 Duroc boars to GWA. We aimed to analyze population-based associations and identify SNPs and QTLs associated with the ADG of pigs. We utilized Illumina Porcine SNP60 BeadChip as the genotyping platform and the mixed linear model (MLM) and multi-locus random-SNP-effect mixed linear model (mrMLM) for GWA [16].

\section{MATERIALS AND METHODS}

\section{Ethic statement}

The experimental procedures used in this study met the guidelines of the Animal Care and Use Committee of the South China Agricultural University (SCAU) (Guangzhou, People's Republic of China). All animal experiments in this study were approved by the Animal Care and Use Committee of the SCAU with the approval number SCAU\#0017.

\section{Animals and phenotypes}

A total of 390 male Duroc pigs were procured from Guangdong Wen's Foodstuffs Co., Ltd. (Guangdong, China). The pigs were born over the period of 2011 to 2014. They were randomly assigned to groups and housed in half-open cementfloor pens. Each pen contained 10 animals, with an average floor space of $2 \mathrm{~m}^{2}$ per pig. The ADG phenotype was identified using Osborne FIRE Pig Performance Testing System (Kansas, NE, USA) by the personnel of Guangdong Wen's Foodstuffs Group Co., Ltd. (Guangdong, China) when the pigs weighed from $30 \mathrm{~kg}$ to $100 \mathrm{~kg}$. All individuals were fed under the same standard conditions with the same feedstuff. Ear tissue was collected as follows: the pig's ear was first cleaned with $75 \%$ alcohol. Then, a clear forfex was used to cut out a small fraction of ear tissue. The wound was then treated with tincture of iodine. The protocol for ear tissue collection was approved by the ethics committee of SCAU.

\section{DNA isolation, genotyping, and quality control}

DNA was extracted from each sample of ear tissue following the standard phenol/chloroform extraction method. All DNA samples were qualified and normalized to a final concentration of $50 \mathrm{ng} / \mu \mathrm{L}$. DNA quality was assessed on the basis of the ratios of light absorption (A260/280 and A260/230) and electrophoresis. The quality and concentration of each genomic DNA sample met the requirements for the Illumina SNP genotyping platform. The 390 individuals were genotyped using the porcine SNP60K Beadchip of Illumina (San Diego, CA, USA) [12]. Quality control was performed using PLINK v 1.07 software [17]. SNP markers with genotype missing rates $>0.05$, call rate $<95 \%$, minor allele frequencies $<0.01$, and Hardy-Weinberg $\mathrm{p} \leq 10 \mathrm{E}-06$ were excluded. Unmapped SNPS and SNPs located on sex chromosomes were removed in accordance with the Sus scrofa 10.2 assembly of the reference genome [18]. Samples and SNPs that passed the filter were selected for subsequent GWA analysis.

\section{Generalized mixed linear model and population stratification assay}

Association analyses based on the generalized MLM were conducted using GenABEL in R software [15]. This model included a random polygenic effect, and the variance-covariance matrix was proportionate to genome-wide identity-bystate [19]. The formula of the model is given by the mathematical expression $Y \sim \mu+\boldsymbol{X} b+K w+S c+Z a+e$. In this expression, $Y$ is the vector of phenotypes; $\mu$ is the overall mean; $b$ is the vector of fixed effects, including yearly and seasonal effects; $w$ is the vector of the body weight of individuals and is considered as a covariate; and $c$ is the vector of SNP effects. In addition, $a$ is the vector of random additive genetic effects with $\mathrm{a} \sim \mathrm{N}(0$, $\mathbf{G} \sigma_{\alpha}^{2}$ ), where $\mathbf{G}$ is the genomic relationship matrix calculated from the corrected pedigree and $\sigma_{a}^{2}$ is the polygenetic additive variance. Finally, $K$ is the regression coefficient of slaughter weight, and $e$ is the vector of residual errors with $\mathrm{e} \sim \mathrm{N}\left(0, \mathbf{I}_{e}{ }_{e}^{2}\right)$, where $I$ is the identity matrix and $\sigma_{e}^{2}$ is the residual variance. $X, S$, and $Z$ are incidence matrices for $b, c$, and $a$, respectively.

Significant thresholds were identified through the Bonferroni method, in which the conventional p-value was divided 
by the number of performed tests [20]. A SNP was considered to have stringent genome-wide significance at $\mathrm{p}<0.05 / \mathrm{N}$ and suggestive significance at $\mathrm{p}<1 / \mathrm{N}$, where $\mathrm{N}$ stands for the number of SNPs tested in the analyses. In this study, the significant and suggestive thresholds were 1.20E-6 (0.05/35971) and $2.40 \mathrm{E}-5$ (1/35971), respectively.

Population stratification can affect the reliability of GWA results. The quantile-quantile (Q-Q) plot is a suitable tool for assessing the presence of population stratification. It is used to examine the distribution of test statistics generated from thousands of association tests and assessing their deviation from the null distribution (the distribution expected under the null hypothesis of no SNP associated with the trait) [21]. In the Q-Q plot (Figure 1A), the horizontal axis and the vertical axis represent the expected $-\log _{10} P$ and the observed $-\log _{10} P$, respectively. The diagonal line represents $y=x$, and the shaded region represents the $95 \%$ confidence interval based on $\beta$-distribution. Overall deviation above the diagonal identity line may indicate severe population stratification [22]. Deviations from the diagonal line suggest that either the assumed distribution is incorrect or that the sample contains values that arose in some other manner, as in a true association [23]. In the present study, the Q-Q plot was generated using R software.

Haplotype or linkage disequilibrium (LD) block analyses

A

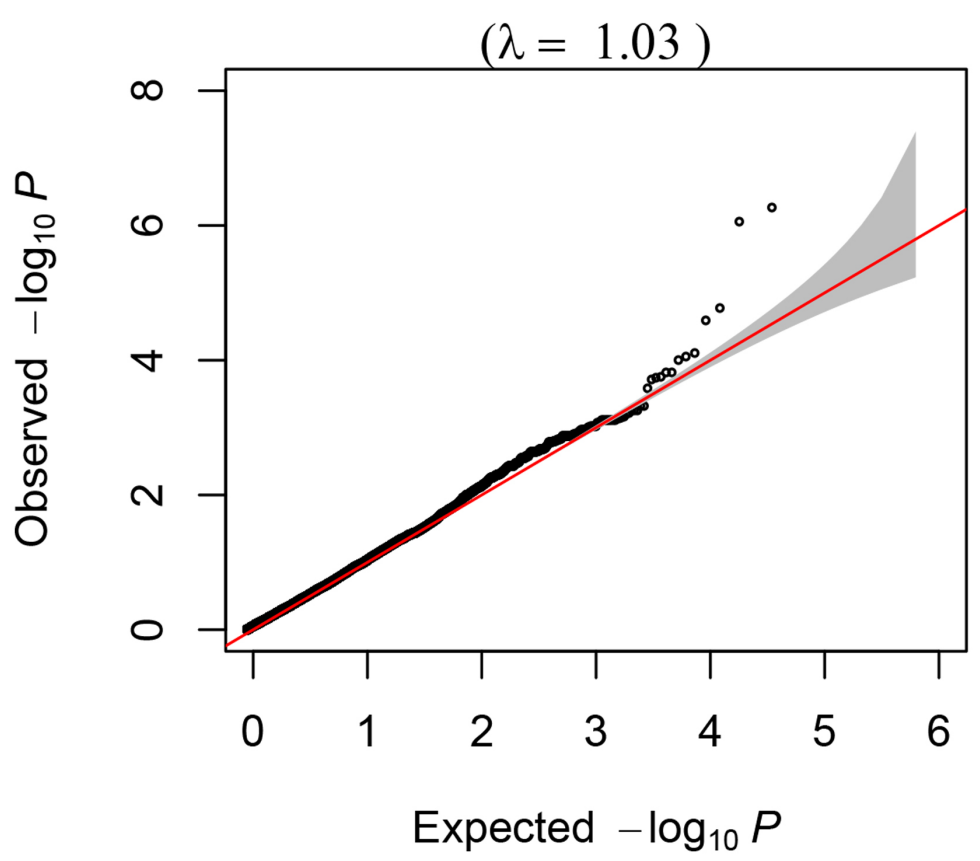

B

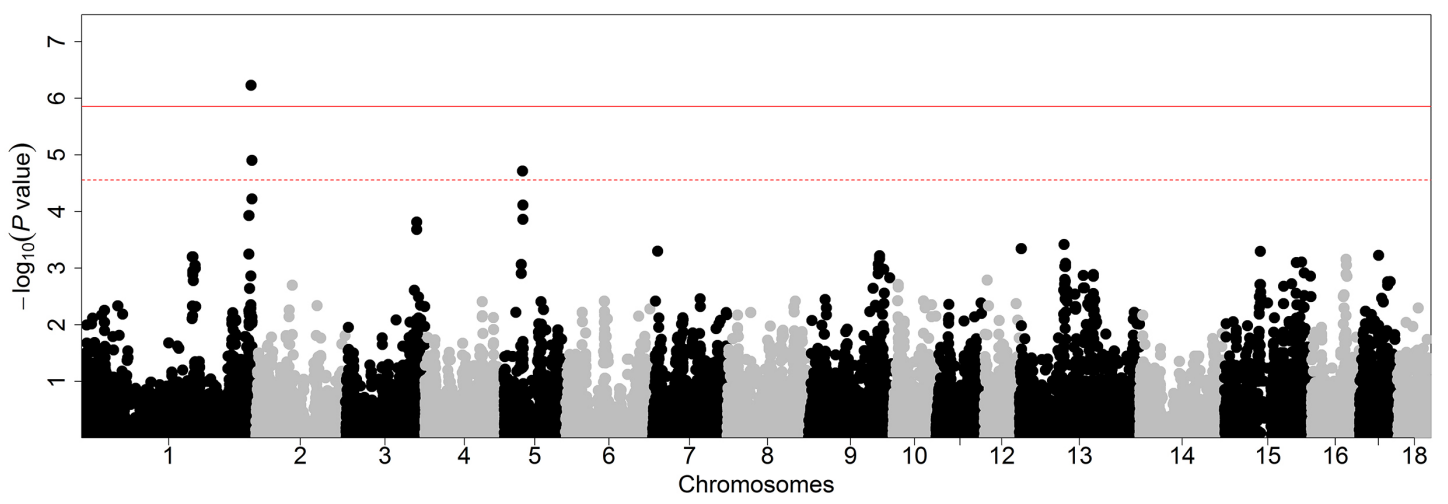

Figure 1. Quantile-quantile and Manhattan plots. (A) Quantile-quantile (Q-Q) plots showing the observed versus expected log p-values for the average daily gain (ADG) trait. The horizontal axis indicates the expected $2 \log _{10}$ (p-values) and the vertical axis indicates the observed $2 \log _{10}$ ( $p$-values). The diagonal line represents $y=x$, which corresponds to the null hypothesis, and the shaded region shows $95 \%$ confidence interval based on $\beta$-distribution. (B) Manhattan plot showing the significance of association between 35,791 single nucleotide polymorphisms (SNPs) and the ADG trait. In the Manhattan plots, the negative $\log _{10}$ p-values of the quantified SNPs were plotted against their genomic positions. The solid and dashed lines indicate the 5\% genome-wide and chromosome-wide Bonferroni-corrected thresholds, respectively. 
were performed for chromosomal regions with multiple significant SNPs clustered around the peak SNP. PLINK v1.07 and Haploview version 4.2 were used to complete haplotype blocks. Then, two candidate genes that were located in the regions of the haplotype block were selected in accordance with functional information retrieved from the Ensembl database (www.ensembl.org) [24,25].

\section{Multi-locus random-SNP-effect mixed linear model}

Common GWA methods are based on single marker analysis and fixed-SNP-effect MLM [14]. These methods are limited by the over-strict correction (Bonferroni correction) of multiple tests. Thus, the genotypic information of potential SNP loci cannot be efficiently used. The mrMLM treats the effect of SNPs as random and allows the modified Bonferroni correction to be used for calculating the threshold p-value for significance tests [16]. GWA based on mrMLM was employed using the R package "mrMLM." We set the significant threshold of log of odds (LOD) score as 4 in our study in accordance with that in Wang et al [26].

\section{RESULTS}

\section{Summary of phenotypic and SNP data}

Table 1 shows the phenotype values of the ADG of experimental pigs. The data in the table include the number of individuals, as well as the mean, standard deviation, maximum, and minimum ADG values. After filtering for quality control, 750 SNPs on sex chromosomes, 5,252 unmapped SNPs, 840 markers that failed the HWE test, 489 SNPs that failed the missingness test, and 18,792 SNPs that failed the frequency test were excluded. A total of 35,971 SNPs $(35,971 / 61,565)$ were used for subsequent analysis (Supplementary Table S1). Supplementary Table $\mathrm{S} 1$ shows that the average marker density (physical distance) of adjacent SNPs on the same chromosome was approximately $0.06 \mathrm{Mb}$ and ranged from $0.05 \mathrm{Mb}$ (Sus scrofa chromosome [SSC]14) to $0.08 \mathrm{Mb}$ (SSC1).

\section{Assessment of population stratification}

Population stratification is a major threat to the reliability of the GWA results [21]. The Q-Q plots of test statistics in the
Table 1. Description of ADG of the studied Duroc population based on phenotype statistics

\begin{tabular}{lccccc}
\hline Traits & N & Mean & SD & Min & Max \\
\hline ADG & 390 & 956.15 & 153.61 & 541.5 & 1,371 \\
\hline
\end{tabular}

$A D G$, average daily gain; $S D$, standard deviation; Min, minimum; Max, maximum.

GWA are shown in Figure 1A. The plot reveals that the ADG data lack clear overall systematic bias. In this study, the average genomic inflation factor $(\lambda)$ of GWA for ADG was 1.03, suggesting the absence of population stratification.

\section{Genome-wide association study}

GWA results through generalized MLM are illustrated in Figure $1 \mathrm{~B}$ and Table 2 . The $\mathrm{p}$-values of (in terms of $-\log _{10} \mathrm{P}$ ) the profiles of all SNPs tested for association with the ADG trait are shown in Figure 1B. Table 2 shows the genome-wide and chromosome-wide significant SNPs for ADG. Only one genome-wide significant (significant) SNP (MARC0025058) and two chromosome-wide significant (suggestive) SNPs (MARC 0074154, H3GA0016186) were identified. Then, two significant SNPs (MARC0025058, H3GA0016186) were mapped to two haplotype blocks spanning 433 and $774 \mathrm{~Kb}$ (Figure 2A). Information about Sus scrofa10.2 retrieved from the Ensembl database revealed that 12 genes (DEAD-box helicase 31, general transcription factor 3C subunit 4, ENSSSCG00000005732, ENSSSCG00000005733, ENSSSCG00000005735, ral guanine nucleotide dissociation stimulator, carboxyl ester lipase, general transcription factor 3C subunit 5 [GTF3C5], ENSSSCG 00000027595, ENSSSCG00000022250, ENSSSCG00000023474, and GFI1B) are located in a block on SSC1. Eight genes, including high mobility group AT-hook 2 (HMGA2), LLP homolog, long-term synaptic facilitation, ENSSSCG00000000473, ENSS SCG00000000474, interleukin 1 receptor associated kinase 3, ENSSSCG00000000476, DNA helicase B, and ENSSSCG0000 0018696, were identified in a block on SSC5 after further analysis. The functional and locational information of the identified genes were retrieved from the Ensembl database and showed that in the haplotype blocks, GTF3C5 and HMGA2 stood out as candidate genes that may be associated with ADG. Through the pig genome database, we found that marker MARC0025058 is on the seventh intron of GTF3C5 on SSC1. Marker H3GA

Table 2. Location of significant SNPs and candidate genes for ADG

\begin{tabular}{|c|c|c|c|c|c|c|c|}
\hline Trait & SNP ID & SSC & ${\text { Location }(b p)^{1)}}^{1)}$ & Adjusted p-value & Candidate gene & Distance $(b p)^{2)}$ & Relative function \\
\hline \multirow[t]{3}{*}{$A D G$} & MARC0025058 & 1 & $306,869,183$ & $5.88 \mathrm{E}-07$ & $\begin{array}{c}\text { GTF3C5 } \\
\text { (ENSSSCG0000000573) }\end{array}$ & within & Skeletal muscle cell differentiation \\
\hline & MARC0074154 & 1 & $308,379,812$ & $1.71 \mathrm{E}-05$ & - & - & - \\
\hline & H3GA0016186 & 5 & $33,594,793$ & $1.93 \mathrm{E}-05$ & HMGA2 & $(+) 78423$ & diet-induced obesity \\
\hline
\end{tabular}

SNP, single nucleotide polymorphism; ADG, average daily gain; SSC, Sus scrofa chromosome; GTF3C5, general transcription factor IIIC subunit 5; HMGA2, high mobility group AT-hook 2 .

1) Derived from the current porcine genome sequence assembly (Sus scrofa10.2).

2) $(+)$ : SNP located in the upstream region of the candidate gene. 
A

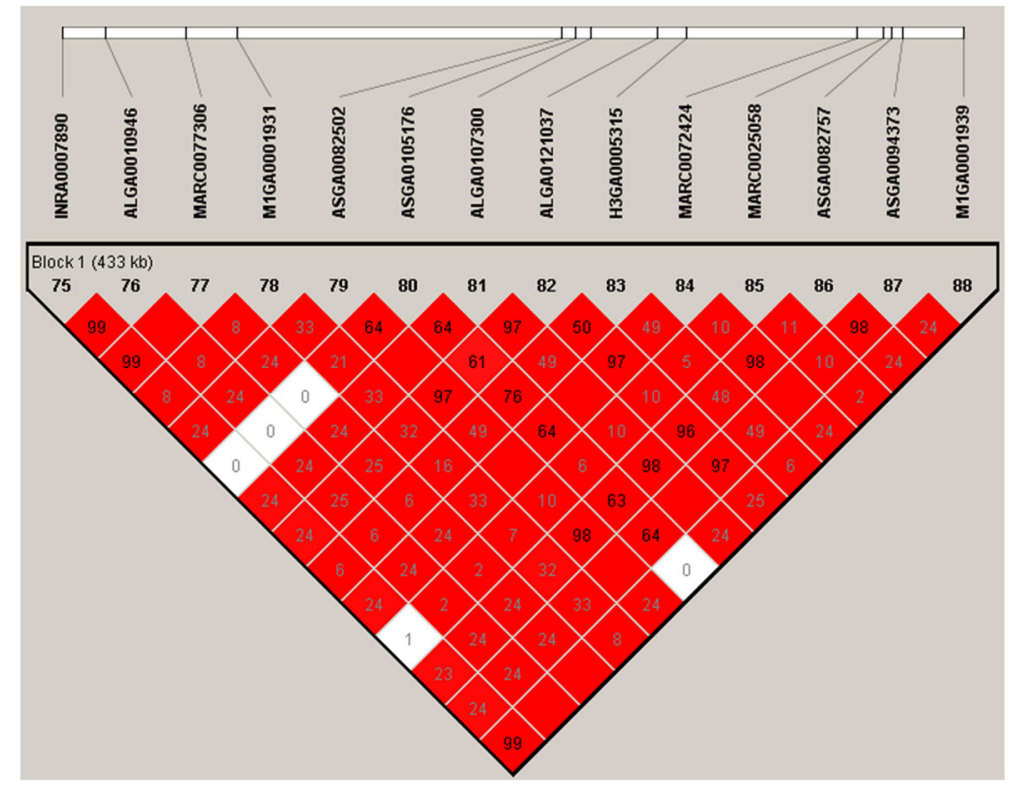

B

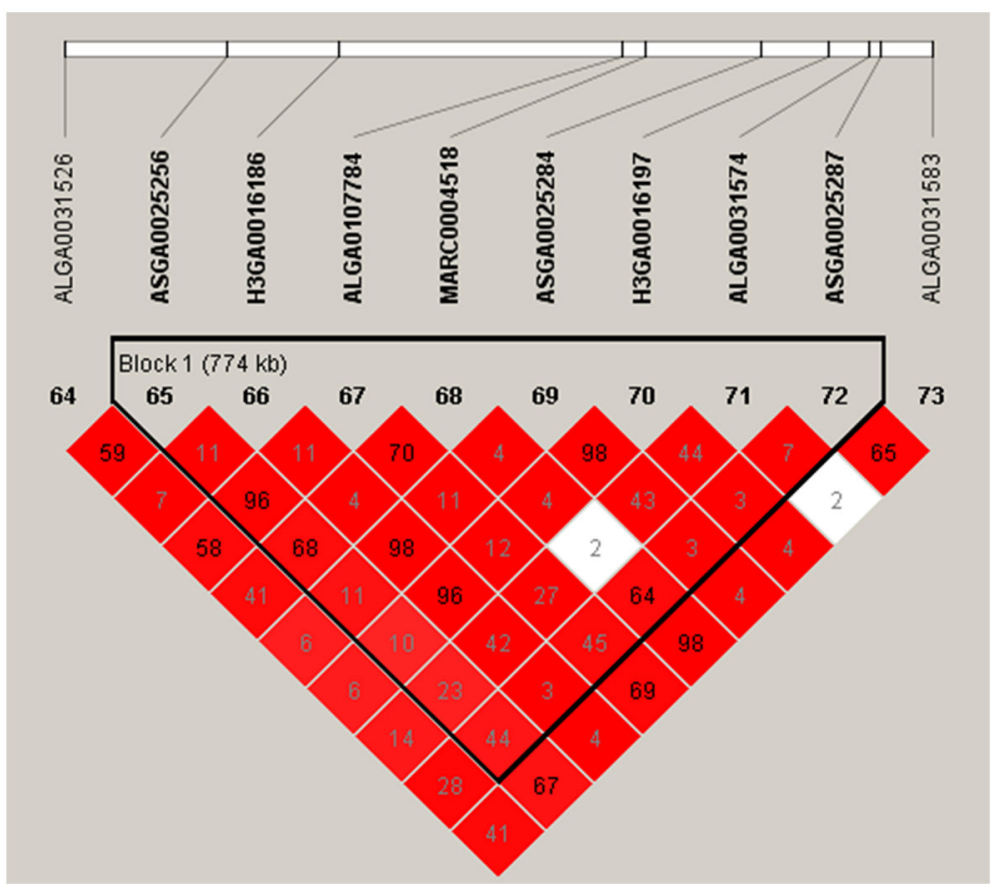

Figure 2. Linkage disequilibrium blocks (LD) on Sus scrofa chromosome (SSC) 1 and SSC5. (A) Linkage disequilibrium blocks on SSC1. (B) Linkage disequilibrium blocks on SSC5. LD blocks are marked with triangles. Values in boxes are the LD (r2) between SNP pairs. The boxes are colored in accordance with the standard Haploview color scheme: $L O D>2$ and $D^{\prime}=1$, red; $L O D<2$ and $D^{\prime}<1$, white ( $L O D$ is the log of the likelihood odds ratio, a measure of confidence in the value of $D^{\prime}$ ).

0016186 is located 78,423 bp upstream of HMGA2 on SSC5. The location of other genes in haplotype blocks are shown in Supplementary Table S2.

GWA results obtained through mrMLM are illustrated in Supplementary Figure S1 and Table S3. All significant SNPs with LOD scores greater than four are shown in Supplemen- tary Figure S1, and the detailed information of these SNPs are shown in Supplementary Table S3. DRGA0006936, DRGA000 9666, INRA0041583, and ASGA0074790 were found on SSC 6, SSC 9, SSC 13, and SSC 16, respectively, and are all associated with ADG. Six candidate genes (GTF3C5, HMGA2, nicotinamide phosphoribosyltransferase [NAMPT], oligodendrocyte 
transcription factor 1 [OLIG1], pleckstrin homology and Rho GEF domain containing G4B [PLEKHG4B], and ENSSSCG 00000031548) were selected based on the information from the Sus scrofa 10.2 database of Ensembl.

\section{DISCUSSION}

\section{Duroc population and GWA}

Duroc pigs can be terminal sire of DLY, which is the most popular commercial pig breed. Improving the growth performance traits, such as ADG, of Duroc is crucial for the pig industry. Thus, identifying the QTL and major genes responsible for the ADG of Duroc is necessary to facilitate the future molecular breeding of commercial pig breeds. GWA provides an efficient method to search for ADG-related genes in pigs. GWA studies are commonly based on single marker analysis and fixed-SNPeffect MLM [14]. These methods, however, are limited by the over-strict Bonferroni correction of multiple tests. Thus, the genotypic information of potential SNP loci cannot be efficiently used. In this study, the mrMLM was employed to estimate the association results produced by MLM based on single-SNP tests and to efficiently use the phenotypic and genotypic information obtained from an enormous amount of accessions and SNPs. Results showed that the two GWA models have the same effectiveness for discovering significant ADG-associated SNPs. However, the GWA based on mrMLM discovered additional SNPs that potentially affect the performance of ADG.

\section{Comparison of QTLs identified in this study with previous studies}

Since Risch et al [11] first proposed the concept of GWA in 1996, it has been a useful method for investigating major genes or diminishing the regions of QTLs associated with specific phenotypes. We utilized two methods to perform the GWA of 390 Duroc individuals. We identified seven SNPs that are significantly associated with the ADG of pigs. Five SNPs on SSC1, SSC5, SSC9, and SSC13 are located in previously reported QTL regions that are associated with the ADG of pigs [27-30]. In the same QTL region of SSC1, Meng et al [31] and Fontanesi et al [32] discovered the H3GA0004299 and ALGA 0009614 SNPs that are associated with the ADG of American Yorkshire pigs and Large Italian White pigs, respectively. In the same QTL region of SSC5, Fontanesi et al [32] discovered four SNPs (M1GA0007246, M1GA0007255, M1GA0007258, and M1GA0007286) that are associated with ADG. The results of previous studies suggested that the QTL associated with ADG might have similar locations in different pig breeds. Howard et al [33], however, found that the QTL span from 176.19 $\mathrm{Mb}$ to $177.76 \mathrm{Mb}$ on SSC1 and that from $84.01 \mathrm{Mb}$ to $84.74 \mathrm{Mb}$ on SSC 5 are associated with ADG in Duroc boars. This result is consistent with previous reports that genes for quantitative traits, such as ADG, are distributed on numerous chromosomal regions of pigs [34]. Moreover, we found two other SNPs DRGA0006936 on SSC6 and ASGA0074790 on SSC16 that have not been included in any previously reported QTLs. The nearest genes of these two SNPs are ENSSSCG00 000031548 and PLEKHG4B. Although the functions of the ENSSSCG00000031548 and PLEKHG4B genes are poorly elucidated, previous studies have reported that the latter is associated with the development of the human brain and nervous system [35]. Results indicated that two QTLs might be associated with the ADG trait in the pig population.

\section{Candidate genes}

In this study, seven SNPs that are significantly associated with the ADG trait were detected on SSC1, SSC5, SSC6, SSC9, SSC13, and SSC16. Six candidate genes that are located close to significant or suggestive SNPs were considered as important candidate genes on the basis of functional and locational information. Functional gene analysis revealed that these candidate genes are mainly involved in skeletal muscle cell differentiation, diet-induced obesity and nervous system development. This result is consistent with that of a previous study [36]. After alignment the sequence of the GTF3C5 gene (http://blast.ncbi. nlm.nih.gov/Blast.cgi), we found that swine GTF3C5 exhibited over $85 \%$ and $81 \%$ homology with human and mouse GTF3C5, respectively. This result indicated that the functions of GTF3C5 in these three species are highly similar. Additionally, information from the UniProt database (http://www.uniprot.org/ uniprot/F1SOS0) revealed that the GTF3C5 gene is indispensable in skeletal muscle cell differentiation in pigs. In humans, the GTF3C5 gene mediates the general protein transcription factor 3C polypeptide $5[37,38]$. The function of GTF3C5 has been studied in mice, and results showed that individuals that are homozygous for GTF3C5 are abnormal and those that are recessive for the gene are non-viable [39]. This result suggested that this gene has crucial roles in embryonic development and in postnatal growth and development. Thus, functional studies on this gene are necessary.

The HMGA2 gene encodes small, chromatin-associated protein high-mobility group AT-hook 2, which belongs to the non-histone chromosomal high-mobility group A family of DNA-binding proteins [40]. This protein can modulate transcription and promote or inhibit the action of transcriptional enhancers by altering chromatin structure or by facilitating the assembly of the multiprotein complexes of transcriptional factors [41-43]. Previous studies have revealed that HMGA2 is highly expressed during embryogenesis; this expression pattern indicates its crucial role in growth and development [44-46]. Knocking out the mouse counterpart of HMGA2 demonstrated that this gene is involved in diet-induced obesity [47]. Given that murine and porcine $H M G A 2$ genes are highly homologous, HMGA2 may have a similar function 
associated with ADG in pigs. Furthermore, previous studies have confirmed that HMGA2 is overexpressed in malignant and benign tumors and is associated with certain characteristic cancer-promoting mutations [48,49]. Notably, HMGA2 is related to height [50,51]. All of the above described functions are indirectly or directly related to weight gain. The majority of functional studies on HMGA2 have focused on its role in human or mouse systems. Additional studies on porcine HMGA2 should be conducted in the future, not only because this gene is related to weight gain, height, carcinogenesis, and tumorigenesis, but also because pigs can be useful animal models for human growth or disease.

Studies on human and mouse systems have shown that NAMPT genes can encode the enzyme nicotinamide 5-phosphoribosyl-1-pyrophosphate transferase, which catalyzes the rate-limiting step in nicotinamide adenine dinucleotide biosynthesis, and that NAMPT is preferentially secreted by visceral fat tissue [52]. Cepica et al [53] found that this gene is associated with the muscling, growth, fat deposition, and fat-tomeat ratio of pigs in the wild boar×Meishan F2 family. In combination with our findings, this gene might be an important candidate for molecular breeding studies that aim to improve the ADG of pigs. OLIG1 and PLEKHG4B genes are associated with the development of the human brain and nervous system and may also encode for neurotropic factors [35,54].

Future studies are necessary to understand or prove the mechanism that underlies the effects of the selected candidate genes on ADG. Future research could include gene sequencing and the identification of mutations, additional statistical association testing, and cell experiments to allow the comparison of differences or associations between mutant and normal cell lines.

\section{CONCLUSION}

The present GWA study was based on two methods and identified seven SNPs that are significantly associated with the ADG of pigs. Six QTL regions for ADG were identified, including four previously identified QTLs on SSC 1, SSC5, SSC9, and SSC13 and two novel QTLs on SSC6 and SSC16. Six candidate genes were singled out on the basis of their functional annotations, positions, and expression patterns in related tissues. The present findings will contribute to the further identification of the casual mutation underlying these QTLs and the future improvement of ADG by pig breeding programs.

\section{CONFLICT OF INTEREST}

We certify that there is no conflict of interest with any financial organization regarding the material discussed in the manuscript. Yang M, Cai G, Wu Z are employees of Guangdong Wens Foodstuffs Co., Ltd..

\section{ACKNOWLEDGMENTS}

This work was supported by grants from the Science and Technology Planning Project of Guangdong Province (2015B020 231010, 2017B020201012), the Natural Science Foundation of China (31601912), and the Natural Science Foundation of Guangdong Province (2016A030310447). The authors are thankful to Guangdong Wen's Foodstuffs Co., Ltd., for phenotype measurements and other contributions to our study.

\section{REFERENCES}

1. Xu X, Xing S, Du ZQ, et al. Porcine TEF1 and RTEF1: molecular characterization and association analyses with growth traits. Comp Biochem Physiol B Biochem Mol Biol 2008;150: 447-53.

2. Liu G, Jennen DG, Tholen E, et al. A genome scan reveals QTL for growth, fatness, leanness and meat quality in a DurocPietrain resource population. Anim Genet 2007;38:241-52.

3. Edwards DB, Ernst CW, Tempelman RJ, et al. Quantitative trait loci mapping in an F2 Duroc $x$ Pietrain resource population: I. Growth traits. J Anim Sci 2008;86:241-53.

4. Murani E, Muraniova M, Ponsuksili S, Schellander K, Wimmers $\mathrm{K}$. Molecular characterization and evidencing of the porcine $\mathrm{CRH}$ gene as a functional-positional candidate for growth and body composition. Biochem Biophys Res Commun 2006;342: 394-405.

5. Harmegnies N, Davin F, De Smet S, et al. Results of a wholegenome quantitative trait locus scan for growth, carcass composition and meat quality in a porcine four-way cross. Anim Genet 2006;37:543-53.

6. Hidalgo AM, Lopes MS, Harlizius B, Bastiaansen JW. Genomewide association study reveals regions associated with gestation length in two pig populations. Anim Genet 2016;47:223-6.

7. Ai H, Huang L, Ren J. Genetic diversity, linkage disequilibrium and selection signatures in chinese and Western pigs revealed by genome-wide SNP markers. PLoS One 2013;8:e56001.

8. Ma J, Yang J, Zhou L, et al. Genome-wide association study of meat quality traits in a White DurocxErhualian F2 intercross and Chinese Sutai pigs. PLoS One 2013;8:e64047.

9. Andersson L. Genome-wide association analysis in domestic animals: a powerful approach for genetic dissection of trait loci. Genetica 2009;136:341-9.

10.Ding R, Quan J, Yang M, et al. Genome-wide association analysis reveals genetic loci and candidate genes for feeding behavior and eating efficiency in Duroc boars. PLoS One 2017; 12:e0183244.

11. Risch N, Merikangas K. The future of genetic studies of complex human diseases. Science 1996;273:1516-7.

12. Ramos AM, Crooijmans RP, Affara NA, et al. Design of a high density SNP genotyping assay in the pig using SNPs identified and characterized by next generation sequencing technology. 
PLoS One 2009; 4:e6524.

13. Edea Z, Hong JK, Jung JH, et al. Detecting selection signatures between Duroc and Duroc synthetic pig populations using high-density SNP chip. Anim Genet 2017;48:473-7.

14. Wang H, Xu C, Liu X, et al. Development of a multiple-hybrid population for genome-wide association studies: theoretical consideration and genetic mapping of flowering traits in maize. Sci Rep 2017;7:40239.

15. Aulchenko YS, Ripke S, Isaacs A, van Duijn CM. GenABEL: an R library for genome-wide association analysis. Bioinformatics 2007;23:1294-6.

16. Wang SB, Feng JY, Ren WL, et al. Improving power and accuracy of genome-wide association studies via a multi-locus mixed linear model methodology. Sci Rep 2016;6:19444.

17. Purcell S, Neale B, Todd-Brown K, et al. PLINK: a tool set for whole-genome association and population-based linkage analyses. Am J Hum Genet 2007;81:559-75.

18. Goedbloed DJ, Megens HJ, Van Hooft P, et al. Genome-wide single nucleotide polymorphism analysis reveals recent genetic introgression from domestic pigs into Northwest European wild boar populations. Mol Ecol 2013;22:856-66.

19. Hayes BJ, Goddard ME. Technical note: prediction of breeding values using marker-derived relationship matrices. J Anim Sci 2008;86:2089-92.

20. Yang Q, Cui J, Chazaro I, Cupples LA, Demissie S. Power and type I error rate of false discovery rate approaches in genomewide association studies. BMC Genet 2005;6 Suppl 1:S134.

21.Pearson TA, Manolio TA. How to interpret a genome-wide association study. JAMA 2008;299:1335-44.

22.Xiong X, Liu X, Zhou L, et al. Genome-wide association analysis reveals genetic loci and candidate genes for meat quality traits in Chinese Laiwu pigs. Mamm Genome 2015;26:181-90.

23. Burton PR Clayton DG, Cardon LR, et al. Genome-wide association study of 14,000 cases of seven common diseases and 3,000 shared controls. Nature 2007;447:661-78.

24. Gabriel SB, Schaffner SF, Nguyen H, et al. The structure of haplotype blocks in the human genome. Science 2002;296: 2225-9.

25. Barrett JC, Fry B, Maller J, Daly MJ. Haploview: analysis and visualization of LD and haplotype maps. Bioinformatics 2005; 21:263-5.

26. Wang N, Chen B, Xu K, et al. Association mapping of flowering time QTLs and insight into their contributions to rapeseed growth habits. Front Plant Sci 2016;7:338.

27. de Koning DJ, Harlizius B, Rattink AP, et al. Detection and characterization of quantitative trait loci for meat quality traits in pigs. J Anim Sci 2001;79:2812-9.

28. Quintanilla R, Milan D, Bidanel JP. A further look at quantitative trait loci affecting growth and fatness in a cross between Meishan and Large White pig populations. Genet Sel Evol 2002;34:193-210.

29. Duthie C, Simm G, Doeschl-Wilson A, et al. Quantitative trait loci for chemical body composition traits in pigs and their positional associations with body tissues, growth and feed intake. Anim Genet 2008;39:130-40.

30.Liu G, Kim JJ, Jonas E, et al. Combined line-cross and half-sib QTL analysis in Duroc-Pietrain population. Mamm Genome 2008;19:429-38.

31.Meng Q, Wang K, Liu X, et al. Identification of growth trait related genes in a Yorkshire purebred pig population by genomewide association studies. Asian-Australas J Anim Sci 2017;30: 462-9.

32. Fontanesi L, Schiavo G, Galimberti G, Calo DG, Russo V. A genomewide association study for average daily gain in Italian Large White pigs. J Anim Sci 2014;92:1385-94.

33. Howard JT, Jiao S, Tiezzi F, et al. Genome-wide association study on legendre random regression coefficients for the growth and feed intake trajectory on Duroc Boars. BMC Genet 2015; 16:59.

34.Hu ZL, Park CA, Wu XL, Reecy JM. Animal QTLdb: an improved database tool for livestock animal QTL/association data dissemination in the post-genome era. Nucleic Acids Res 2013;41:D871-9.

35. Raffield LM, Cox AJ, Hugenschmidt CE, et al. Heritability and genetic association analysis of neuroimaging measures in the Diabetes Heart Study. Neurobiol Aging 2015;36:1602.e7-15.

36. Wang K, Liu D, Hernandez-Sanchez J, et al. Genome wide association analysis reveals new production trait genes in a male Duroc population. PLoS One 2015;10:e0139207.

37.Hsieh YJ, Wang Z, Kovelman R, Roeder RG. Cloning and characterization of two evolutionarily conserved subunits (TFIIIC102 and TFIIIC63) of human TFIIIC and their involvement in functional interactions with TFIIIB and RNA polymerase III. Mol Cell Biol 1999;19:4944-52.

38. Lander ES, Linton LM, Birren B, et al. Initial sequencing and analysis of the human genome. Nature 2001;409:860-921.

39. Gardin A, White J. The sanger mouse genetics programme: high throughput characterisation of knockout mice. Acta Ophthalmol 2010 Sept 23 [Epub] https:/doi.org/10.1111/ j.1755-3768.2010.4142.x

40.Ashar HR, Tkachenko A, Shah P, Chada K. HMGA2 is expressed in an allele-specific manner in human lipomas. Cancer Genet Cytogenet 2003;143:160-8.

41. Falvo JV, Thanos D, Maniatis T. Reversal of intrinsic DNA bends in the IFN beta gene enhancer by transcription factors and the architectural protein HMG I(Y). Cell 1995;83:1101-11.

42. Reeves R. Molecular biology of HMGA proteins: hubs of nuclear function. Gene 2001;277:63-81.

43. Shi Z, Wu D, Tang R, et al. Silencing of HMGA2 promotes apoptosis and inhibits migration and invasion of prostate cancer cells. J Biosci 2016;41:229-36.

44.Zhou X, Benson KF, Ashar HR, Chada K. Mutation responsible for the mouse pygmy phenotype in the developmentally regulated factor HMGI-C. Nature 1995;376:771-4. 
45. Chiappetta G, Avantaggiato V, Visconti R, et al. High level expression of the HMGI (Y) gene during embryonic development. Oncogene 1996;13:2439-46.

46.Zhou X, Chada K. HMGI family proteins: architectural transcription factors in mammalian development and cancer. Keio J Med 1998;47:73-7.

47.Federico A, Forzati F, Esposito F, et al. Hmga1/Hmga2 double knock-out mice display a "superpygmy" phenotype. Biol Open 2014;3:372-8.

48. Bol S, Wanschura S, Thode B, et al. An endometrial polyp with a rearrangement of HMGI-C underlying a complex cytogenetic rearrangement involving chromosomes 2 and 12. Cancer Genet Cytogenet 1996;90:88-90.

49. Fusco A, Fedele M. Roles of HMGA proteins in cancer. Nat Rev Cancer 2007;7:899-910.
50. Hodge JC, Cuenco KT, Huyck KL, et al. Uterine leiomyomata and decreased height: a common HMGA2 predisposition allele. Hum Genet 2009;125:257-63.

51. Yang TL, Guo Y, Zhang LS, et al. HMGA2 is confirmed to be associated with human adult height. Ann Hum Genet 2010; 74:11-6.

52.Imai S. Nicotinamide phosphoribosyltransferase (NAMPT): a link between NAD biology, metabolism, and diseases. Curr Pharm Des 2009;15:20-8.

53. Cepica S, Bartenschlager H, Ovilo C, et al. Porcine NAMPT gene: search for polymorphism, mapping and association studies. Anim Genet 2010;41:646-51.

54.Prasad A, Teh DBL, Blasiak A, et al. Static magnetic field stimulation enhances oligodendrocyte differentiation and secretion of neurotrophic factors. Sci Rep 2017;7:6743. 